\title{
Thermal Design and In Situ Temperature Measurement of Heterogeneous Material during Ultrafast Laser Scribing
}

\author{
Wen-Fei Lin, ${ }^{1}$ Hsiang-Chen Hsu, ${ }^{2,3,4 *}$ Shih-Jeh Wu, ${ }^{4}$ and Boen Houng ${ }^{1}$ \\ ${ }^{1}$ Department of Material Science and Engineering, I-Shou University, \\ No. 1, Sec. 1, Syuecheng Rd., Dashu District, Kaohsiung City 84001, Taiwan, ROC \\ ${ }^{2}$ Department of Mechanical and Computer-Aided Engineering, St. John's University, \\ No. 499, Sec. 4, Tam King Rd., Tamsui District, New Taipei City 25135, Taiwan, ROC \\ ${ }^{3}$ Department of Industrial Management, I-Shou University, \\ No. 1, Sec. 1, Syuecheng Rd., Dashu District, Kaohsiung City 84001, Taiwan, ROC \\ ${ }^{4}$ Department of Mechanical and Automation Engineering, I-Shou University, \\ No. 1, Sec. 1, Syuecheng Rd., Dashu District, Kaohsiung City 84001, Taiwan, ROC
}

(Received March 26, 2018; accepted April 3, 2019)

Keywords: in situ temperature, ultrafast laser, heterogeneous material, surface temperature distributions

In this paper, an in situ technique is developed to measure the temperature distribution during the ultrafast laser scribing (machining) of heterogeneous materials using embedded sensors. The materials investigated in this study are an epoxy molding compound (EMC), solder bumps, and a specially designed ultrathin printed circuit board (PCB). The laser machining processes involved in this study are cutting/scribing through EMC and $\mathrm{PCB}$, and trenching/scribing through EMC alone. Small thermocouples have been designed and inserted inside the heterogeneous materials to record the in situ temperature distribution during ultrafast laser irradiation and the thermal effect is carefully investigated. The highest temperature of $100.94{ }^{\circ} \mathrm{C}$ is recorded at the corner scribing position. For comparison, a forward-looking infrared (FLIR) thermal imaging camera was applied to capture the real-time surface temperature distributions of PCB and EMC. Owing to the ambient heat radiation, material emissivity, and reflective apparent temperature, the peak surface temperature detected by FLIR would be slightly lower than the in situ temperature measured by an embedded sensor. The thermal design in this jam-packed area is then applied to a standard laser manufacturing process.

\section{Introduction}

The epoxy molding compound (EMC) has been widely applied to the IC packaging material in the semiconductor industry for decades. ${ }^{(1-3)}$ EMC consists of 20 raw materials, such as epoxy resin (organic resin), fused silica (inorganic filler), catalysts, mold release material, pigment, flame retardants, adhesion promoters, ion traps, and stress relievers. ${ }^{(1)}$ Usually, epoxy and filler dominate the mechanical property of EMC, that is, $12-15 \mathrm{wt} \%$ epoxy resin provides good machining quality and $70-80 \mathrm{wt} \%$ filler increases the strength and thermal conduction, *Corresponding author: e-mail: hchsu@isu.edu.tw https://doi.org/10.18494/SAM.2019.2328 
and reduces the coefficient of thermal expansion. After the components on the printed circuit board (PCB) are encapsulated by EMC and singulated as one single unit, the whole package of heterogeneous materials undergoes a burn-in test process at the early stage of the mass product period. This test purposely induces certain failures under stringent conditions, such as that of the end product, and determines the reliability of the end product. ${ }^{(4,5)}$

Traditionally, saw cutting is the main solution to singulate the integrated circuit unit. As new technology innovation emerges, there are new challenges; for example, in cutting irregular shapes and rigid and flexible materials, a mechanical saw can no longer meet the requirement, and a laser has become a new solution for singulation. ${ }^{(6)}$ As a mechanical saw has to be operated in cutting fluid media, a laser works with air similarly. However, that is no comparison to the heat effect related to product reliability. ${ }^{(7)}$ For instance, laser scribing creates a warmer environment than a normal burn-in temperature, i.e., $250{ }^{\circ} \mathrm{C}$ in most cases, and a regular burn-in test should be exercised under a more rigorous condition in the cases related to the laser process. There will be more laser applications to come during the assembly of systemin-package $(\mathrm{SiP})$ products. More applications need more components in a regular PCB area, which would result in the undesired crosstalk of signals. SiP is a design with high-density components on $\mathrm{PCB}$, so high-giga components need to be isolated to prevent the noise effect on other components by shielding. ${ }^{(8)}$ The specific structure of SiP shielding in a compound, which is hard to build by molding, can be created by laser scribing. ${ }^{(9)}$

It is interesting to researchers and engineers to investigate how to maintain the performance of the components shielded in the package after the laser machining of the shielding structure. High temperature causes defects on the cured epoxy, such as material melting, deterioration, cracks, and loss of adhesion to copper. ${ }^{(10)}$ Using a short or ultrashort pulse laser is a solution for semiconductor processes. ${ }^{(11)}$ The main reason for this is that they cause a minimal thermal effect during the scribing processes. Those reasons explain why both researchers and engineers are interested in how high the temperature is during laser scribing. In this study, the authors present the design of the in situ temperature measurement of heterogeneous materials during ultrafast laser scribing. A noncontact forward-looking infrared (FLIR) thermal imaging camera is capable of scanning and visualizing the temperature distribution of an entire surface in many industrial applications..$^{(12,13)}$ However, there is still some difficulty encountered when FLIR is detecting the temperature of the surface during laser machining. Thus, the use of K-type thermocouples inserted inside the material near laser scribing has been demonstrated to be an accurate approach to measure the temperature in situ.

\section{Materials and Methods}

A nanosecond UV laser (355 nm wavelength, $20 \mathrm{~W}$ power, $<1.3 \mathrm{M} 2$ beam quality, $200 \mu \mathrm{J}$ pulse energy, and 90-110 kHz base frequency) was used, and the optimal machining parameters for the cutting and trenching of the molding compound and PCB of SiP were explored. In laser scribing, the target material is a mixture of heterogeneous materials of EMC and PCB. The epoxy compound with a thickness of $0.8 \mathrm{~mm}$ in an ultrafine filler size range of $15-35 \mu \mathrm{m}$ and PCB with a thickness of $0.36 \mathrm{~mm}$ were tested to investigate thermal behaviors during laser 
scribing. There are four copper layers in $\mathrm{PCB}$, where three copper layers are in the cutting street and the one copper layer is solder-printed for trenching. Each copper layer is $0.5 \mathrm{oz}$, and between EMC and PCB, there is a solder of about $45 \mu \mathrm{m}$ height printed on the copper pad for the trenching dump, as shown in Fig. 1. Note that a solder microball has been deformed by laser trenching.

\subsection{Optics system}

The original laser beam is insufficient for cutting through a SiP structure and needs optics to support the scribing process. A galvo-scanner head was used for high-feed-rate scribing, and a telecentric lens was used for a more vertical wall side, which was installed with a laser. Figure 2(a) schematically illustrates an innovated optical installation developed in this study. ${ }^{(14)}$ A focal shifter helps in the installation, which easily makes the beam focus on the material bottom and is fixed during scribing. The theoretical spot size is designed to be $13.7 \mu \mathrm{m}$ for this system.

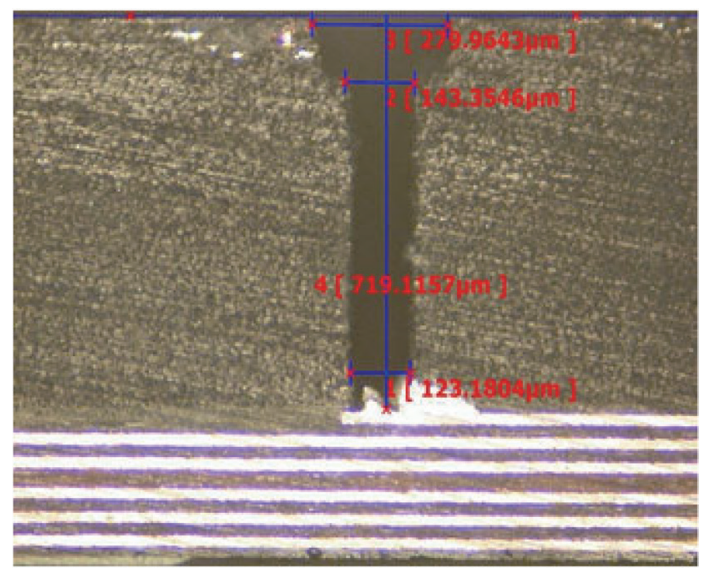

Fig. 1. (Color online) Cross section of SiP material.

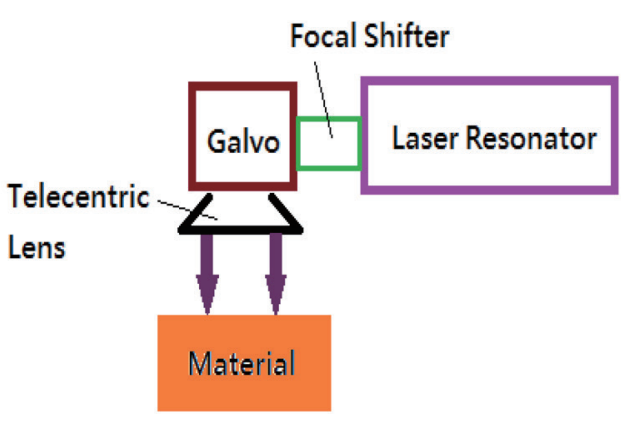

(a)

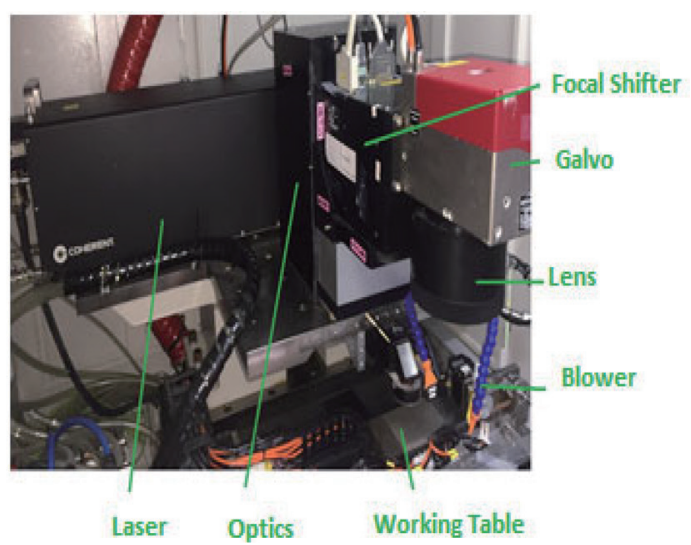

(b)

Fig. 2. (Color online) (a) Schematic illustration of optics design system. (b) Actual installation inside the laser machine. 
Figure 2(b) shows the actual optics system used in this study. First, a laser beam is generated by the laser resonator. The beam was manipulated by a focal shifter and a galvo to scribe on the position designed on the material, which will be described in Sect. 2.3. The telecentric lens makes the beam a small spot with a machine ability on the material.

\subsection{Laser trench/cutting in scribing process}

For trenching, the parameters are as follows: a feed speed of $800 \mathrm{~mm} / \mathrm{s}$, a repetition rate of $100 \mathrm{kHz}$, and an output average power of $17.5 \mathrm{~W}$ measured under the lens. The scribing process used 146 passes to create the trench, which is shown in Fig. 3.

For the cutting process, the parameters measured under the lens are as follows: a feed speed of $800 \mathrm{~mm} / \mathrm{s}$, a repetition rate of $100 \mathrm{kHz}$, and an output average power of $17.5 \mathrm{~W}$. The scribing process was repeated 100 times to cut through the material.

After trenching and removing the material, the top view of the test sample is shown in Fig. 4. The cutting shape is a rectangle and the trenching shape is a turned $\mathrm{L}$ shape from the top view of the SiP. The total cutting length is $108 \mathrm{~mm}$ with four corners, and the total trenching length is $14 \mathrm{~mm}$ with one corner. Note again that there are three copper layers in the cutting street and one more copper layer in the trenching position for solder-printed trenching.

\subsection{In situ temperature measurement}

K-type thermocouples were used for in situ measurement in this study. The SiP was drilled with some 1-mm-diameter holes and then thermocouples were inserted into the holes. All the holes were drilled from PCB side with different depths as shown in Fig. 5, i.e., (a) a sensor inserted into the middle of EMC, (b) a sensor inserted into the middle of PCB, and (c) a sensor inserted into the middle of a solder bump.

Note that the K-type thermocouple is the most common sensor calibration type providing the widest operating temperature range and generally works in most applications. A thermocouple

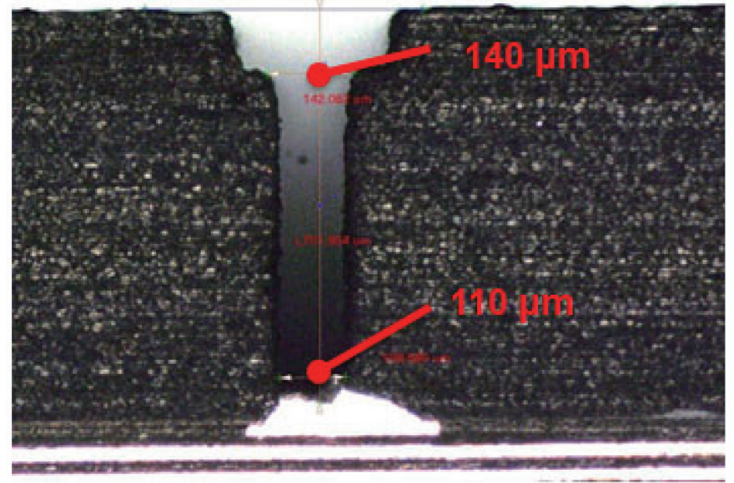

Fig. 3. (Color online) Cross section of trenching structure designed in SiP.

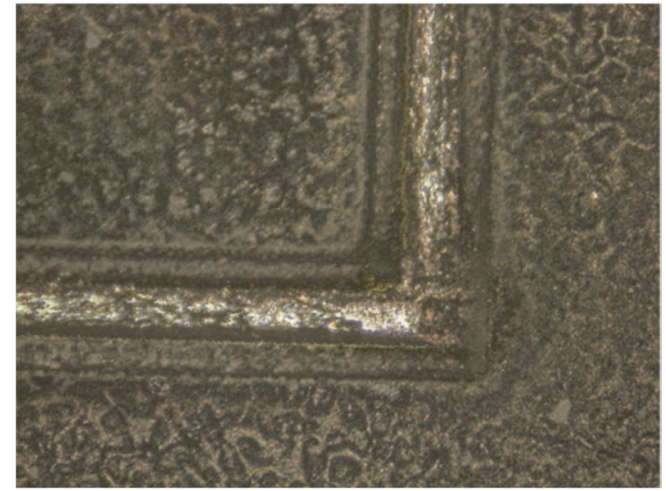

Fig. 4. (Color online) Top view of test sample for scribing (trenching and cutting). 


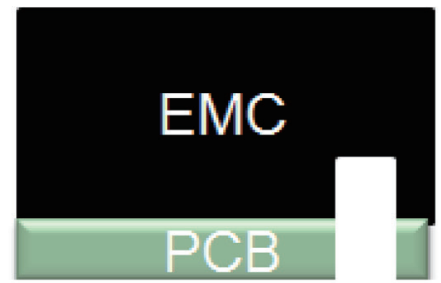

(a)

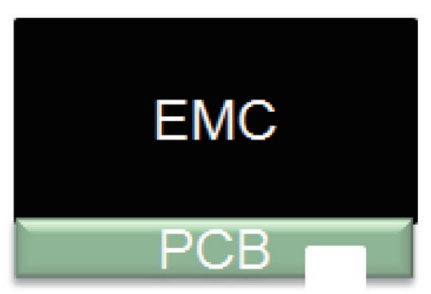

(b)

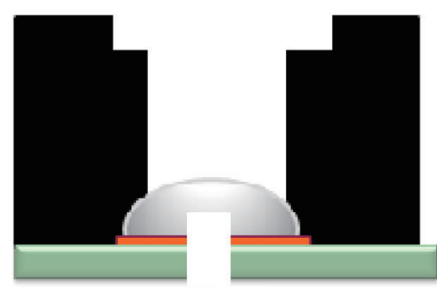

(c)

Fig. 5. (Color online) Cross sections of drilling holes with different depths for a thermocouple to measure (a) EMC, (b) $\mathrm{PCB}$, and (c) solder bump.

circuit contains two alloy junctions, such as wire sand connectors and a voltage-measuring device. When two different temperatures were detected, measurable current flows through the circuit. The current is related to the temperature differential, which presents the detected temperature. Normally, the output signals from the K-type thermocouple are very small and need to be carefully calibrated before use. Owing to its reliability and accuracy, the $\mathrm{K}$-type thermocouple is used extensively at temperatures up to $1260{ }^{\circ} \mathrm{C}$. However, the K-type thermocouple with a temperature range of $0-275^{\circ} \mathrm{C}$ was used in this study.

For EMC and PCB temperature measurements, the in situ temperature was assessed for both cutting and trench processes, respectively. For solder temperature measurement, the in situ temperature was assessed only for the trench process. The thermocouples' positions were gauged, as shown in Fig. 6, and designed $200 \mu \mathrm{m}$ away from the scribing area, which is a normal position where an electronic component is located. For the temperature measured in a printed solder bump, Fig. 6(c) illustrates the thermocouples measured for the trenching process only, which were only inserted at the trenching position.

There would be four thermocouples inserted in four different holes, labeled T1 to T4 in Fig. 6(a), to collect temperature data at the same time in one cutting process, and three thermocouples in three different holes, labeled T1 to T3 in Fig. 6(b), to collect temperature data at the same time in one trenching process. The positions of three thermocouples used to collect the in situ temperature data of the solder in the trenching process are labeled T1 to T3, as shown in Fig. 6(c).

\subsection{Design of experiment (DOE)}

Three materials (EMC, PCB, and solder), in situ temperature measurement (positions labeled T1 to T5), and two scribing processes (cutting and trenching) were used to create five DOEs, which are summarized in Table 1. Note that there is no temperature measured in the solder bump for the cutting process.

Each DOE was carried out individually, namely, DOE1 to DOE3 show one trench process to obtain three temperatures from three thermocouples for one material, and DOE4 to DOE5 show one cutting process to obtain four temperatures from four thermocouples for one material. 


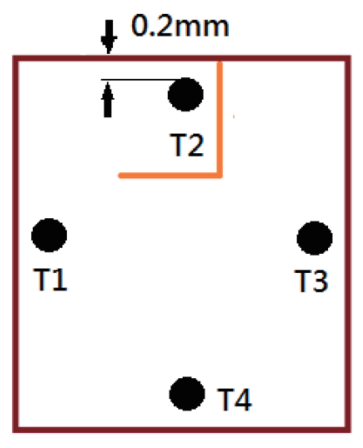

(a)

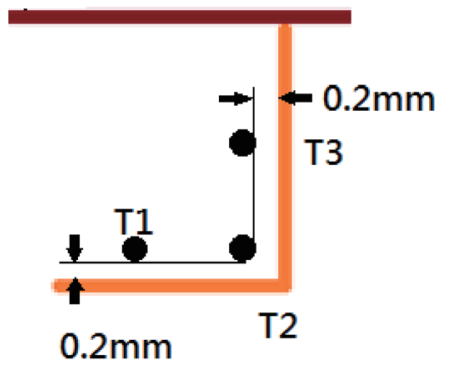

(b)

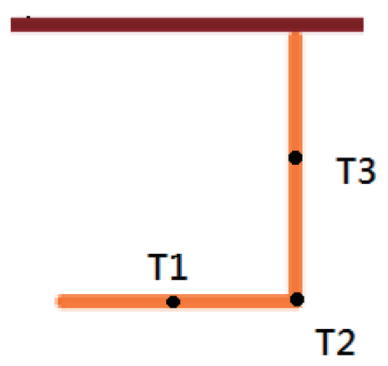

(c)

Fig. 6. (Color online) Schematic diagrams of thermocouples' positions of (a) cutting, (b) trenching, and (c) solder bump in trenching.

Table 1

DOEs used in this study.

\begin{tabular}{lcccccc}
\hline DOE No. & Process & Material & \multicolumn{4}{c}{$\begin{array}{c}\text { Position label of in situ } \\
\text { temperature measurement }\end{array}$} \\
\hline DOE1 & Trench & EMC & T1 & T2 & T3 & - \\
DOE2 & Trench & PCB & T1 & T2 & T3 & - \\
DOE3 & Trench & Solder & T1 & T2 & T3 & - \\
DOE4 & Cutting & EMC & T1 & T2 & T3 & T4 \\
DOE5 & Cutting & PCB & T1 & T2 & T3 & T4 \\
\hline
\end{tabular}

\subsection{Surface temperature real-time detection}

An alternative real-time temperature detected using a FLIR thermal imaging camera was used for comparison. The FLIR thermal imaging camera can distinguish fixed forward-looking thermal images from sideways-tracking infrared systems, which can very easily generate a real-time high-resolution image. A noncontact FLIR thermal imaging camera can scan and visualize the temperature distribution of an entire surface. However, the system requires material emissivity and reflective apparent temperature to precisely detect the temperatures of the target materials.

Figure 7 shows the material emissivity calibration scheme used in this study. The object under test (PCB) was inserted with a K-type thermocouple and placed on a heating plate. The FLIR thermal imaging camera was aimed toward the target PCB with the thermocouple. The real-time temperature was recorded as the heating plate was continuously heating. Once the temperature in the thermal image was exactly the same as that detected by the K-type thermocouple, the material emissivity was then determined using the FLIR thermal imaging camera system. The calibrated material emissivity for PCB is 0.96 . Figure 8 shows the realtime temperature detection equipment used in this study. 


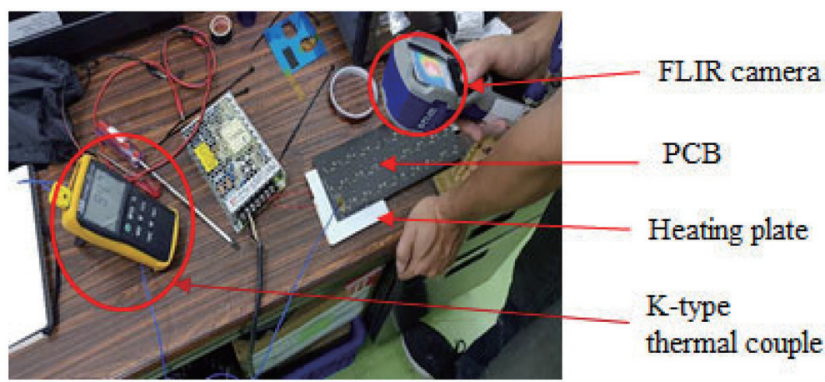

Fig. 7. (Color online) Emissivity calibration for PCB material.

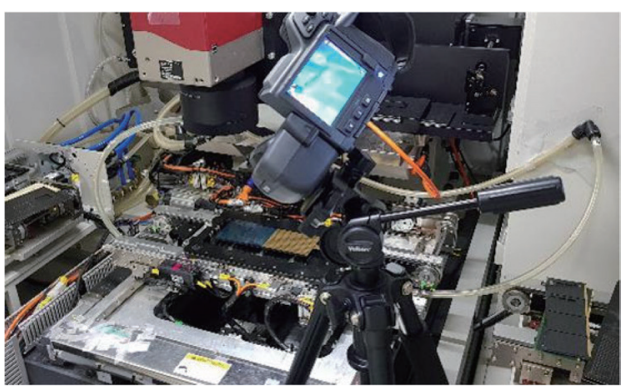

Fig. 8. (Color online) Real-time surface temperature detection using FLIR 440 cameras.

\section{Results and Discussion}

Figure 2(b) shows the main devices for laser machining. The test samples (workpiece) were mounted on an XY table and exposed to the atmosphere (air). At the upper right corner, there is an air blower (nozzle) to remove ash, dust, and residues from the machined surface. The temperature of the laser's front end is assumed to be high enough to melt the workpiece. However, the temperature of the machined surface will be easily transferred to the operating ambient. Hence, the real-time surface temperature measured using the FLIR thermal imaging camera would be relatively lower than the actual laser machining temperature. Therefore, the embedded K-type thermocouple would be appropriate for measuring the in situ temperature along the cut depth.

The thermocouples were connected to a monitor and recorded the accumulated temperature in the sample during laser scribing. It was one cycle with four steps, i.e., start of thermocouple sampling, start of laser scribing, end of laser scribing, and finally, end of thermocouple sampling. The temperature-time chart for each DOE was then automatically graphed. When laser scribing started, the temperature also rose at the same time. The temperature immediately fell after laser scribing. The measured peak temperature for every positioned thermocouple was also reported for the five DOEs. The temperatures measured in situ are shown in Figs. 9-13.

After the 5 DOEs were carried out, the maximum temperature from the thermocouple sampled in one cycle received for all 25 graphs. Results were collected and are listed in Table 2. The obtained temperature variances were as follows: DOE1, $23.62{ }^{\circ} \mathrm{C}$; DOE2, $19.65{ }^{\circ} \mathrm{C}$; DOE3, $32.45{ }^{\circ} \mathrm{C}$; DOE $4,12.60{ }^{\circ} \mathrm{C}$; DOE5, $19.38^{\circ} \mathrm{C}$. This implies that the area near the solder bump would cause a large thermally induced warpage owing to the mismatch of the coefficient of thermal expansion among heterogeneous materials.

For comparison in the cutting process, the FLIR thermal imaging camera system with a thermal imaging system was used to detect the surface real-time temperature distributions of PCB and EMC, which are depicted in Figs. 14(a) and 14(b), respectively. PCB is a typical multilayer material consisting of copper foil, resin matrix, reinforcement, prepreg, and fillings. The temperature captured on PCB may vary owing to laser cutting on a metal or resin. Also, 


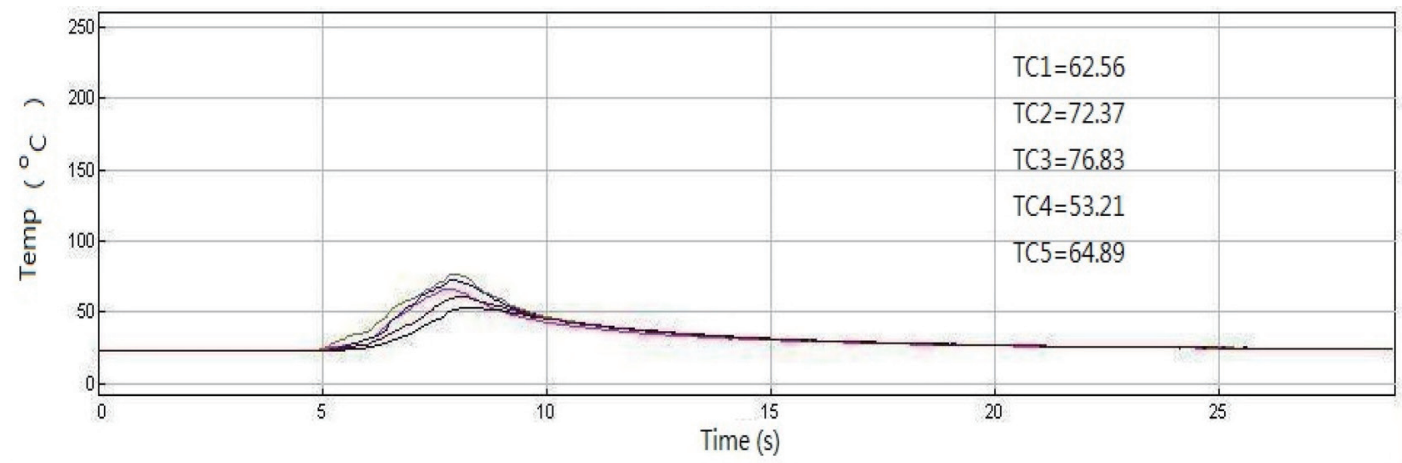

Fig. 9. (Color online) DOE1: temperatures of EMC measured in situ during trenching $\left({ }^{\circ} \mathrm{C}\right)$.

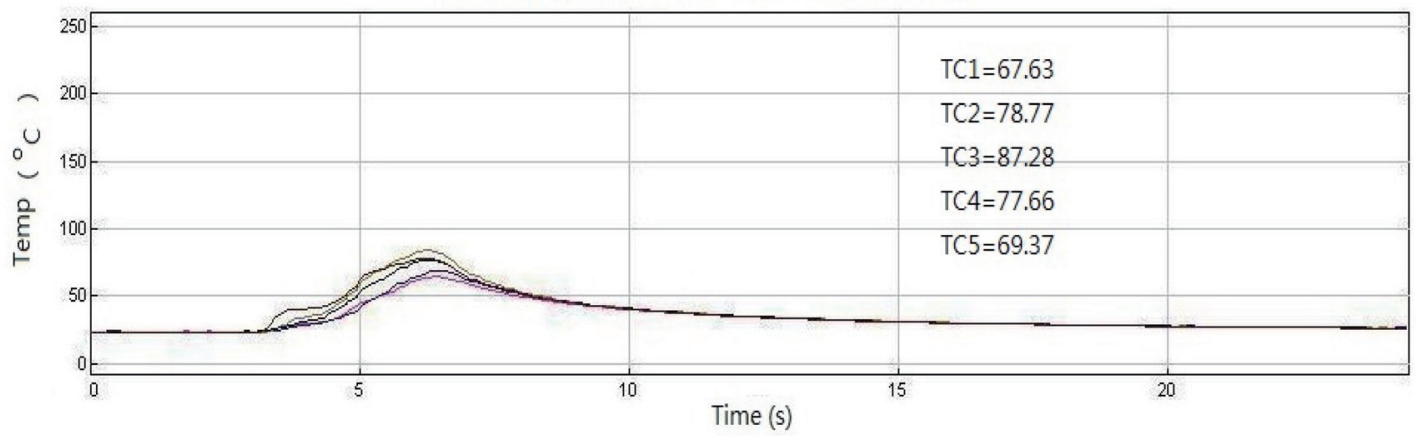

Fig. 10. (Color online) DOE2: temperatures of PCB measured in situ during trenching $\left({ }^{\circ} \mathrm{C}\right)$.

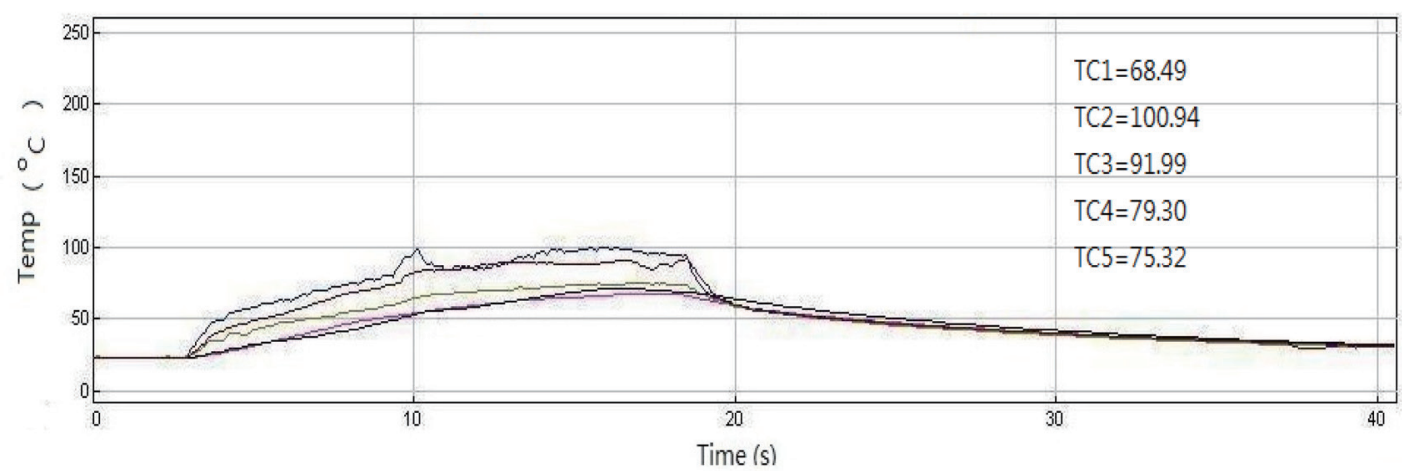

Fig. 11. (Color online) DOE3: temperatures of solder measured in situ during trenching $\left({ }^{\circ} \mathrm{C}\right)$.

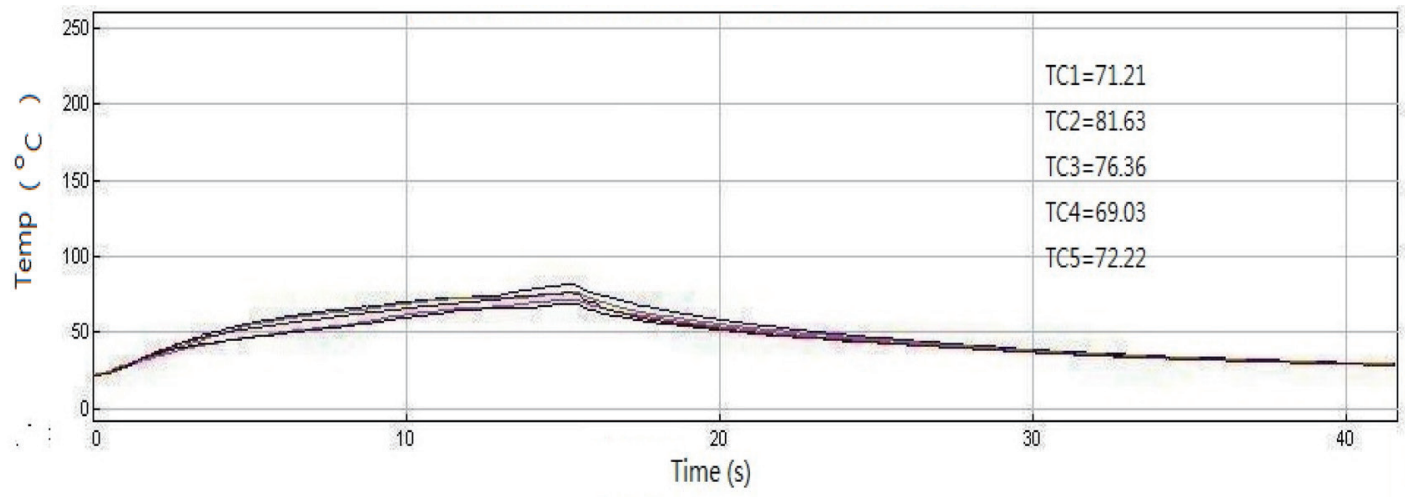

Fig. 12. (Color online) DOE4: temperatures of EMC measured in situ during cutting $\left({ }^{\circ} \mathrm{C}\right)$. 


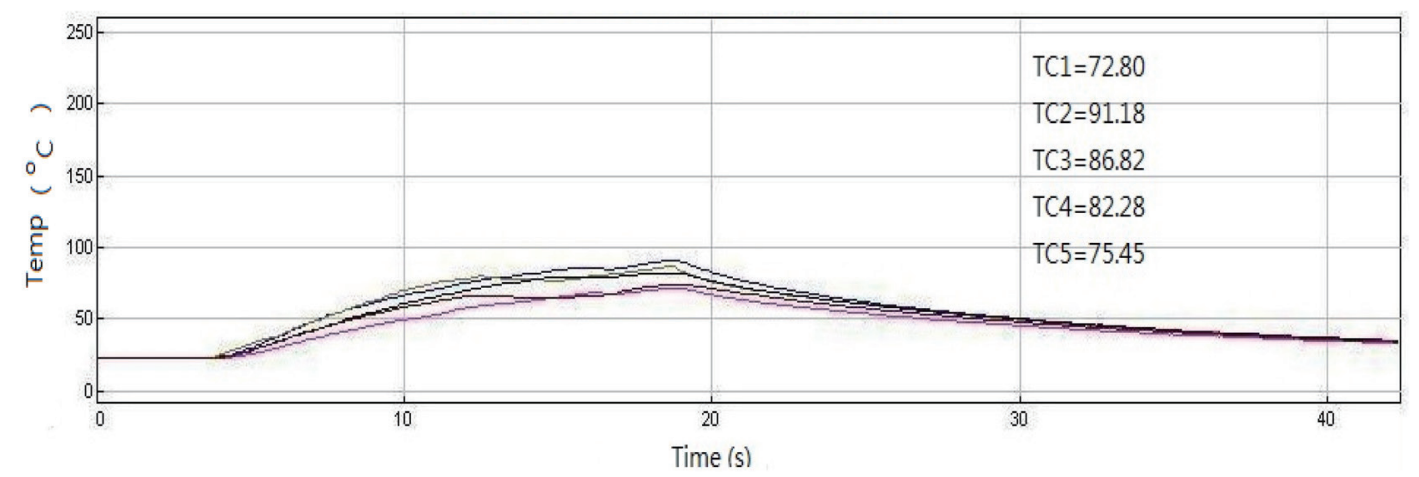

Fig. 13. (Color online) DOE5: temperatures of PBC measured in situ during cutting $\left({ }^{\circ} \mathrm{C}\right)$.

Table 2

In situ temperature of laser scribing (unit: ${ }^{\circ} \mathrm{C}$ ).

\begin{tabular}{lccrrrrc}
\hline \multirow{2}{*}{ DOE No. } & \multirow{2}{*}{ Process } & \multirow{2}{*}{ Material } & \multicolumn{5}{c}{ Position label } \\
\cline { 4 - 8 } & & T1 & T2 & T3 & T4 & T5 \\
\hline DOE1 & Trench & EMC & 62.56 & 72.37 & 63.98 & 53.21 & 64.89 \\
DOE2 & Trench & PCB & 67.63 & 78.77 & 87.28 & 77.66 & 69.37 \\
DOE3 & Trench & Solder & 68.49 & 100.94 & 91.99 & 79.30 & 75.32 \\
DOE4 & Cutting & EMC & 71.21 & 81.63 & 76.36 & 69.03 & 72.22 \\
DOE5 & Cutting & PCB & 72.8 & 91.18 & 86.82 & 82.28 & 75.45 \\
\hline
\end{tabular}

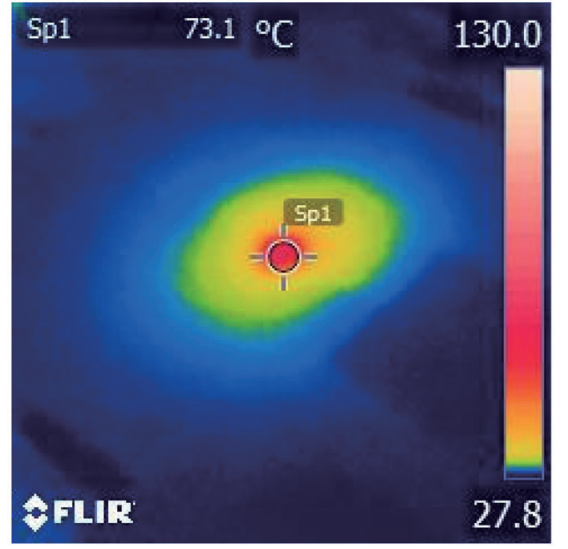

(a)

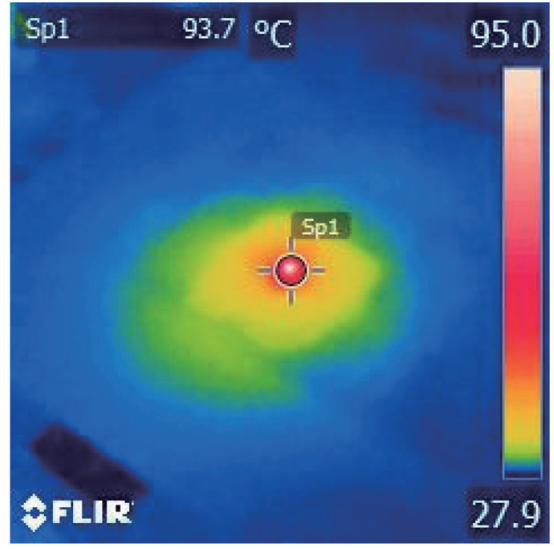

(b)

Fig. 14. (Color online) Cutting temperature distributions captured using FLIR camera system: (a) PCB and (b) EMC.

the in-depth temperature of PCB was difficult to reflect on the surface owing to the various conductivities of the materials. The temperature of EMC is slightly higher than the temperature measured by the thermocouple. This may be caused by the ambient radiation effects on direct laser cutting. 


\section{Conclusions}

In this paper, the authors describe the temperature response of a material and how it was detected by sensors. Engineers would very much like to have more space to mount more components and ICs in a very limited space in PCB.

During cutting, the highest temperature $\mathrm{T} 2$ was observed by $\mathrm{T} 2$ on a trench pad, i.e., $81.63{ }^{\circ} \mathrm{C}$ for EMC and $91.18{ }^{\circ} \mathrm{C}$ for PCB. During the trenching, the highest temperature was obseved at $\mathrm{T} 2$ in the corner, i.e., $72.37^{\circ} \mathrm{C}$ for EMC, $78.77^{\circ} \mathrm{C}$ for $\mathrm{PCB}$, and $100.94{ }^{\circ} \mathrm{C}$ for a solder bump.

During laser cutting, the solder on the trench pad would be the key medium to dissipate more heat to the T2 position shown in Fig. 6(a). During trenchire, heat was spread from two sides to the T2 position shown in Fig. 6(b).

All the temperatures measured in situ during the laser process are lower than the burn-in test temperature and even lower than the curing temperature of EMC. Thermal effects from laser irradiation will not cause any damage to the electronic components under the parameter provided in Sect. 2, i.e., $0.2 \mathrm{~mm}$.

The FLIR thermal imaging camera system is capable of measuring the real-time temperature distributions near the laser cutting surface. However, the system highly depends on the ambient radiation, material emissivity, and reflective apparent temperature. The in situ temperature measurement with embedded thermocouples inside the material developed in this research is much more feasible and reliable.

\section{Acknowledgments}

This work was supported by ASE Kaohsiung, Taiwan and ENR Corporation. The authors thank Bear Jiang and Peter Ku for providing the test materials. We also thank the ENR RD team for providing an integral laser scribing machine.

\section{References}

1 H. C. Hsu, S. J. Wu, W. F. Lin, and B. Houng: Polym. Polym. Compos. 26 (2018) 1.

2 D. E. Lee, H. W. Kim, B. S. Kong, and H. O. Choi: J. Appl. Polym. Sci. 134 (2017) 45252.

3 Y. Huang, D. Bigio, and M. G. Pecht: IEEE Trans. Comp. Packag. Technol. 29 (2006) 364.

4 S. H. Park, J. Y. Park, and T. H. Kim: Electron. Mater. Lett. 9 (2013) 459.

5 J. A. Herbsommer: IEEE Trans. Comp. Packag. Technol. 2 (2012) 1293.

6 A. Alwaidh, M. Sharp, and P. French: Opt. Lasers Eng. 58 (2014) 109.

7 X. C. Wang, Z. L. Li, T. Chen, B. K. Lok, and D. K. Y. Low: Opt. Lasers Eng. 48 (2008) 5.

8 J. M. Yannou, C. Zinck, E. Cheng, V. K. Liao, A. Chan, and N. Tien: Proc. Semicon West (2016).

9 G. B. J. Cadot, D. A. Axinte, and J. Billingham: Int. J. Mach. Tools and Manu. 107 (2016) 8.

10 K. Ahn, S. H. Park, and Y. H. Kim: Microelectron. Reliab. 78 (2017) 1.

11 J. Meijer, K. Du, A. Gillner, D. Hoffmann, V. S. Kovalenko, T. Masuzawa, A. Ostendorf, R. Poprawe, and W. Schulzc: CIRP Ann. 51 (2002) 2.

12 M. Popescu, A. Paino, K. Stone, and J. K. Keller: IEEE Symp. Computational Intelligence for Security and Defence Applications (IEEE, 2012) 1-5.

13 FLIR System: https://www.flir.com/ (accessed July 2018).

14 W. F. Lin and C. H. Chang: ROC Patent No. M510208 (2015). 


\section{About the Authors}

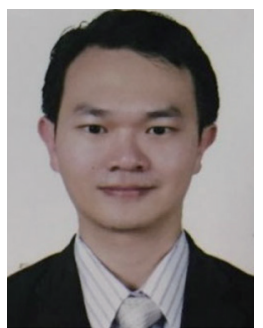

Wen-Fei Lin received his B.S. degree from Chang Gung University, Taiwan, in 1997 and his M.S. degree from National Sun Yat-sen University, Taiwan, in 2000. Currently, he is a Ph.D. candidate of the Department of Material Science and Engineering, I-Shou University. Since 2015, he has been a director of Engineering, Process \& Field Application at E\&R Engineering Corporation, Taiwan. His research interests are in laser machining, material behavior, and assembly.

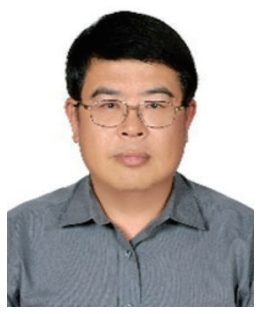

Hsiang-Chen Hsu received his B.S. degree from National Cheng Kung University, Taiwan, in 1981 and his M.S. and Ph.D. degrees from North Carolina State University, USA in 1988 and 1993, respectively. After graduation, he was an associate professor and became a full professor at I-Shou University, Taiwan. Since 2017, he has been temporarily transferred to St. John's University as vice president. His research interests are in IC packaging, reliability design, and automation.

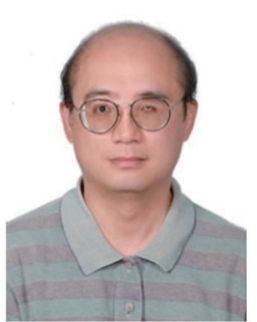

Shih-Jeh Wu received his B.S. degree from National Chen-Kung University, Taiwan, in 1982 and his M.S. and Ph.D. degrees from Pennsylvania State University, USA, in 1995 and 1997, respectively. Since 2000, he has been a faculty member and now an associate professor at I-Shou University, Taiwan. His research interests are in nondestructive evaluation, ultrasonics, laser engineering, bioengineering, and sensory systems.

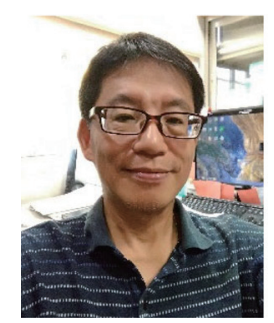

Boen Houng received his B.S. degree from Taipei Institute of Technology, Taiwan, in 1986 and his M.S. and Ph.D. degrees from Drexel University and Colorado School of Mines, USA, in 1991 and 1996, respectively. From 2003 to 2007, he was an assistant professor at I-Shou University, Taiwan. Since 2013, he has been a professor at I-Shou University. His research interests are in thin film technology, oxide ceramics, and electronic materials. 